\title{
Raman Study on Doped Multiwalled Carbon Nanotubes
}

\author{
S. Costa And E. Borowiak-Palen* \\ Centre of Knowledge Based Nanomaterials and Technologies, Institute of Chemical Engineering and Chemical \\ Technology, Westpomeranian University of Technology, Pułaskiego 10, 70-322 Szczecin, Poland
}

\begin{abstract}
In the present study we report resonance Raman study on multiwalled carbon nanotubes treated with $\mathrm{HCl}$, $\mathrm{HNO}_{3}$ and $\mathrm{H}_{2} \mathrm{SO}_{4}$. Acids are commonly used in purification procedures in order to remove catalyst particles from the as-produced material. However, it is known that acid treatments in multiwalled carbon nanotubes can induce a surface functionalisation and intercalation of the walls, modifying their electronic properties. Here, we present a comparative study between samples of multiwalled carbon nanotubes modified with the most common acids used in the purification of carbon materials, and the influence of the acid treatment time on their vibronic properties. The samples were analysed via the Raman spectroscopy using the laser length of $785 \mathrm{~nm}$. The presented data reveal the modification of the typical multiwalled carbon nanotubes Raman bands such as: disorder band ( $\mathrm{D}$ band), the graphite band ( $\mathrm{G}$ band), and the two-phonon second order Raman band $\left(\mathrm{G}^{\prime}\right.$ band). The last one shows a broadening effect on its line shape and the appearance of an additional peak. All the treatments enhance the number of defects in the graphitic structure of multiwalled carbon nanotubes.
\end{abstract}

PACS numbers: 78.67.Ch, 73.22.-f, 81.07.De, 61.72.Ww, 63.20.-e

\section{Introduction}

Carbon nanotubes (CNT) are nowadays very interesting materials due to their large range of applications. CNT are nanostructures derived from rolled graphene planes [1] whose electronic properties can be controlled. One possible way to modify the electronic and vibronic properties is a charge transfer during their intercalation and functionalisation [2-4]. For this reason the doping of CNT has been widely explored to understand the modifications carried out by the guest molecules. One of the most common doping processes is the acid treatment. It is also known that chemical modifications, such as acid treatment can influence the solubility and purity of CNT [5]. Recently, several investigations have been performed on singlewalled (SWCNT) $[6,7]$, doublewalled (DWCNT) $[2-4,8]$ and multiwalled (MWCNT) $[9,10]$ carbon nanotubes involving acid studies. A very interesting study of Graupner et al. [8] on the influence of $\mathrm{H}_{2} \mathrm{SO}_{4}, \mathrm{HNO}_{3}$ and $\mathrm{HCl}$ acids on SWCNT properties was presented. Different studies on MWCNT doped by acids were also reported previously. In particular, doping by nitric acid [9] and by a mixture of $\mathrm{HNO}_{3}$ and $\mathrm{H}_{2} \mathrm{SO}_{4}$ [10] are very interesting. However, to the best of our knowledge the samples of MWCNT treated with the acids have never been analysed by the Raman spectroscopy.

On the other hand, MWCNT with different structural properties can be obtained depending on the preparation method. It is possible to either synthesise "Russian doll-like" structures, or "scroll" nanotubes derived from a single rolled graphene sheet. The intercalation of the MWCNT can be also used to determine the type of tubes

* corresponding author; e-mail: eborowiak@ps.pl
$[11,12]$. Russian doll-type tubes have a reactivity size dependent which can lead to the breaking up of the tubes during the chemical treatment. In the same conditions the scroll tubes preserve their structure [12].

In the current work Raman spectroscopy (excitation laser length $785 \mathrm{~nm}$ ) is used to perform the comparative study on the vibronic and electronic properties of MWCNT treated with three different acids: $\mathrm{H}_{2} \mathrm{SO}_{4}$, $\mathrm{HNO}_{3}$ and $\mathrm{HCl}$. The influence of the acid treatment time on Raman response of MWCNT is also presented.

\section{Experimental}

In this study, MWCNT were prepared by chemical vapour deposition (CVD) technique. The detailed description of the synthesis process has been described elsewhere [13]. The raw material was purified with diluted $\mathrm{HCl}(12 \mathrm{M})$, and then annealed at $600^{\circ} \mathrm{C}$ in high vacuum (ca. $10^{-6}$ mbar), to recover their electronic properties $[3,14]$. Afterwards, the batches of MWCNT were transferred to the three beakers containing the concentrated acid solution of $\mathrm{H}_{2} \mathrm{SO}_{4}$ or $\mathrm{HNO}_{3}$ or $\mathrm{HCl}$, respectively. The acid treatment time was ranging between $0.17 \mathrm{~h}$ and $720 \mathrm{~h}$. Afterwards, the samples were filtrated and washed with water and acetone. The characterisation of all the samples was performed by Raman Spectroscopy (Renishaw InVia Raman microscope) using the laser energy $1.58 \mathrm{eV}(785 \mathrm{~nm})$.

\section{Results and discussion}

The vibronic properties of carbon nanotubes can be modified by the introduction of guest molecules to their structure. Depending on the type of doping (intercalation with electron donors or acceptors), and the dopant 
itself, the Raman response of the system can differ. In this study, we compare the modifications of MWCNT sample treated with three different acid compounds leading to acceptor-type doping, and the influence of the acid treatment time on their properties.

Figure 1a presents the Raman spectra of MWCNT samples doped with the different acids. One can observe two features: the disorder induced mode, the so called $\mathrm{D}$ band (centred at $1300 \mathrm{~cm}^{-1}$ for the reference sample), and the graphite mode, G band (centred at $1589 \mathrm{~cm}^{-1}$ for the reference sample). The well crystallised carbon materials are not affected by the acid [3]. Therefore, a certain number of defects are required in the structure of the CNT to trace the modification of the Raman response upon doping process. Therefore, the analysis of both D band and G band is crucial. These features are strongly dependent on the doping effects which is already known from the study on graphite intercalation compounds [4]. From Fig. 1a, one can observe that the line shape of both features is preserved even after the long time exposure to the acid solution. However, both bands are affected and shifted in the direction of the higher wavelengths in respect of the reference sample. In Fig. 1b one can see the Raman shifts corresponding to the CNT treated with the different acids for the period of time from $0.17 \mathrm{~h}$ to $720 \mathrm{~h}$. The sample treated with $\mathrm{H}_{2} \mathrm{SO}_{4}$ presents higher upshifts in the $\mathrm{D}$ band region (3 to $\left.6 \mathrm{~cm}^{-1}\right)$, while the $\mathrm{G}$ band region is only slightly affected $\left(0\right.$ to $\left.2 \mathrm{~cm}^{-1}\right)$. The $\mathrm{D}$ band of $\mathrm{HNO}_{3}$ treated sample exhibits an even higher upshift $\left(4\right.$ to $\left.8 \mathrm{~cm}^{-1}\right)$. However, in this sample the $\mathrm{G}$ band shifts from 2 to $7 \mathrm{~cm}^{-1}$. For both acid treatments the modifications appear even after short time of treatment (starting from $0.17 \mathrm{~h}$ ). Only in the case of $\mathrm{HCl}$ treatment a dependence of the immersion time of MWCNT in the acid solution is clearly observed. The D band shift of this sample is strongly shifted already after $1 \mathrm{~h}$ of the treatment. However, in order to see the enhanced $\mathrm{G}$ mode upshift a longer period of time is required.

The difference between $\mathrm{HNO}_{3}, \quad \mathrm{H}_{2} \mathrm{SO}_{4}$ treated MWCNT, and $\mathrm{HCl}$ treated MWCNT can be explained by the molecular structure of the used dopants. $\mathrm{H}_{2} \mathrm{SO}_{4}$ and $\mathrm{HNO}_{3}$ contain oxygen atoms in their structure. It is known that oxygen can be easily attached to the defects in the CNT bond network which leads to greater modifications in the defect band (which is currently observed). Graupner et al. [8] also observed that the Raman signal of the metallic tubes of SWCNT in the G band region depends on the exposure to the oxidizing acids. In the case of $\mathrm{HCl}$ treatment (oxygen free molecule), this effect is not so evident and higher upshifts of the $\mathrm{G}$ band can be observed after the longer treatment time. These upshifts are related to a direct electron charge transfer process from the nanotubes to the acceptor molecule [3, 15].

Another aspect which should be considered is the sample quality modification upon the acid treatment. The guest molecule can either intercalate the walls [16], or react with the defects in the surface creating attached

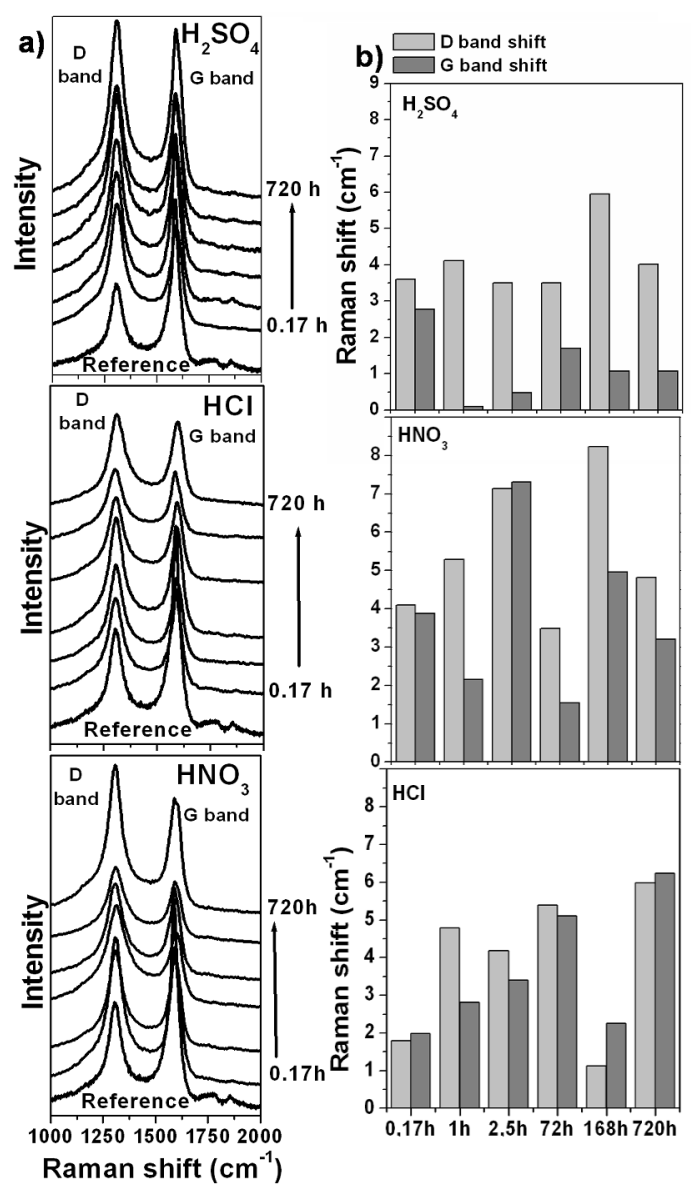

Fig. 1. (a) Raman spectra of the MWCNT treated with the $\mathrm{H}_{2} \mathrm{SO}_{4}, \mathrm{HNO}_{3}$ and $\mathrm{HCl}$ acids. The representation shows two important features in the spectra, the defect mode ( $\mathrm{D}$ band) and the graphite mode ( $\mathrm{G}$ band), while the time of reaction between the CNT and the dopant is changed; (b) shift of the D and G bands on the doped nanotubes compared to the reference sample.

functional groups [5]. It is known that both cases lead to an increase of the amount of defects in the CNT [17]. The $\mathrm{D}$ band indicates the defects in the graphitic structure and the G/D intensity ratio presents the relative sample purity.

In Fig. 2, the curves presenting the G/D intensities ratios versus the acid treatment period of time for the analysed samples, in respect of the reference sample are shown. First, the G/D ratio for $\mathrm{H}_{2} \mathrm{SO}_{4}$ drops drastically already after an $0.17 \mathrm{~h}$ treatment. The trend is changed for the samples treated for $1 \mathrm{~h}$ and the $\mathrm{G} / \mathrm{D}$ ratios slightly increase. The longer treatments result in a gentle decrease of the G/D ratios. Finally, the treatment with $\mathrm{HCl}$ shows a gradual decrease of the $\mathrm{G} / \mathrm{D}$ ratios until $72 \mathrm{~h}$ and a slight increase of it after that period of time. However, in overall the trend for each case is the same, and shows that even short periods of time influence the sample quality significantly. In this respect one can conclude that electron doping via acid treatment 
strongly affects the number of defects in the CNT surface. Additionally, this effect is almost the same for all the analysed samples and no significant acid treatment time dependence is observed.

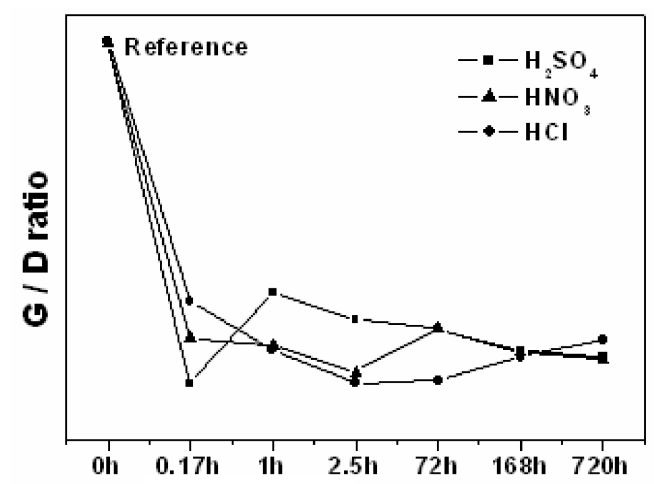

Fig. 2. Relation of $D$ and $G$ bands intensity. The so called $\mathrm{G} / \mathrm{D}$ ratio provides information about the number of defects of the tubes. In the particular case of acid doping, the ratio drops drastically even after a short time of reaction, indicating an intercalation of the host molecules in the tubes.

Next important feature in the Raman spectrum of MWCNT is the $\mathrm{G}^{\prime}$ band originating from a double resonant process [18]. Fagan et al. [19] observed the upshift of this band upon the intercalation of the tubes with the acceptor dopant. On the other hand, Barros et al. [2] detected the $\mathrm{G}^{\prime}$ band expansion upon $\mathrm{H}_{2} \mathrm{SO}_{4}$ intercalation of the tubes and a change on their line shape. A decrease in the intensity of this band was also observed.

In Fig. 3 one can observe the evolution of $\mathrm{G}^{\prime}$ band profile when the acid treatment increases. The $\mathrm{G}^{\prime}$ band position is situated at $2585 \mathrm{~cm}^{-1}$ for the reference sample. Initially, the line shape of the $\mathrm{G}^{\prime}$ band is preserved for all the samples and looks like in the top part of Fig. 3. However, after a certain period of time of immersion in acid the band broadening is observed for each sample. The interesting point is that different treatment time of each material is required to detect this observation: (i) $\mathrm{H}_{2} \mathrm{SO}_{4}$ treated sample $-2.5 \mathrm{~h}$, (ii) $\mathrm{HNO}_{3}$ treated sample $72 \mathrm{~h}$, and (iii) $\mathrm{HCl}$ treated sample $-720 \mathrm{~h}$. In addition to the peak broadening an additional peak appears. This effect is very well pronounced in the case of the $\mathrm{H}_{2} \mathrm{SO}_{4}$ treated nanotubes (see Fig. 3). The diverse $\mathrm{G}^{\prime}$ band profiles observed for the different acid treatments can be explained by the different molecular composition of the guest molecules. The treatments with oxidising acids (acceptors of electrons) provoke stronger charge transfer and consequently the energy of the Fermi level shifts more significantly [7]. A comparison between the $\mathrm{H}_{2} \mathrm{SO}_{4}$ and $\mathrm{HNO}_{3}$ acids doping in SWCNT was performed previously by Zhou et al. [7]. He investigated the electronic properties of $p$-doped SWCNT by temperature-dependent resistivity and thermopower, optical reflectivity, and Raman spectroscopy. It was found that the CNT had a non-

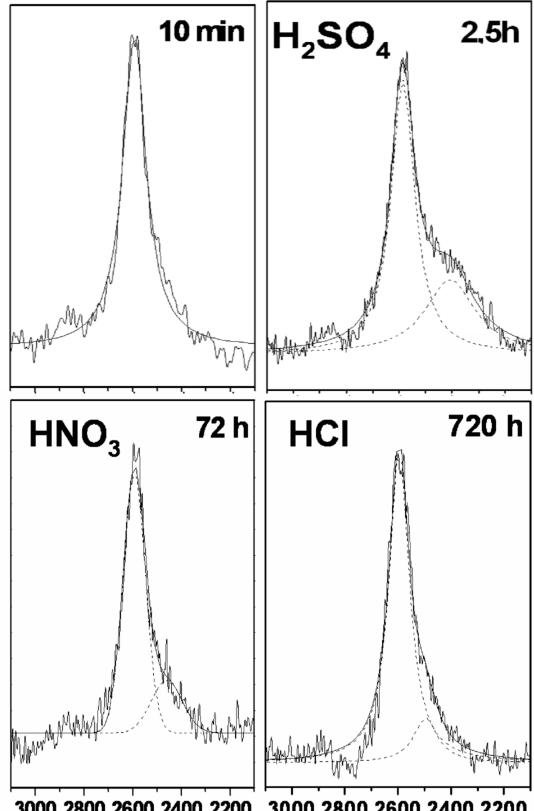

3000280026002400220030002800260024002200 Raman shift $\left(\mathrm{cm}^{-1}\right)$ Raman Shift $\left(\mathrm{cm}^{-1}\right)$

Fig. 3. G band of the Raman spectra of different samples. The band becomes broader after the acid treatment and the appearance of a new peak is observed. The minimum time of acid treatment to observe this fact varies with the different dopant compounds: $\mathrm{H}_{2} \mathrm{SO}_{4}$ $-2.5 \mathrm{~h} ; \mathrm{HNO}_{3}-72 \mathrm{~h} ; \mathrm{HCl}-720 \mathrm{~h}$.

-metallic behaviour before the doping process. For the doped samples there was observed a decrease of the resistivity, and consequently a metallic behaviour was observed. It was also concluded that $\mathrm{H}_{2} \mathrm{SO}_{4}$ is a stronger oxidative dopant than $\mathrm{HNO}_{3}$, since the resistivity of the sample decreased more upon intercalation with $\mathrm{H}_{2} \mathrm{SO}_{4}$. In this study no analysis of the $\mathrm{G}^{\prime}$ band profile of the Raman response was reported. In respect of our data that study could explain the significant modification of $\mathrm{G}^{\prime}$ band upon the charge transfer in MWCNT during the acid treatment. Especially, this effect upon $\mathrm{H}_{2} \mathrm{SO}_{4}$ treatment is well pronounced. In order to confirm this explanation, more Raman studies using different laser energies are required. Hence, further experiments will be performed to get the full understanding of this phenomenon.

\section{Conclusions}

In summary, we studied the Raman response of the acid treated $\left(\mathrm{H}_{2} \mathrm{SO}_{4}, \mathrm{HNO}_{3}, \mathrm{HCl}\right) \mathrm{MWCNT}$ in respect of the pristine sample. It was found that the vibronic and electronic properties of the nanotubes are strongly affected by the acid treatment. The most pronounced modifications of the Raman modes of MWCNT were detected for the samples treated with $\mathrm{H}_{2} \mathrm{SO}_{4}$ and the $\mathrm{HNO}_{3}$. This was due to presence of oxygen in their molecular structures and their stronger interaction with the MWCNT 
structure. The relative purity of the treated MWCNT was also influenced by the introduction of the guest molecules. Even after short time of immersion in acid solution, the number of defects in the graphitic structure of the nanotubes increased significantly in respect of the pristine tubes. It was also found an interesting phenomenon in the $\mathrm{G}^{\prime}$ band region, namely the broadening of the mode and the appearance of the new peak were observed. The origin of this peak in not fully understood so far and further investigations are required.

\section{Acknowledgments}

Research was sponsored by the State Committee for Scientific Research grant 1 T09B 00930 (EB-P) and by the European Community through the Marie Curie Research Training Network CARBIO under contract MRTN-CT-2006-035616.

\section{References}

[1] S. Iijima, T. Ichiashi, Nature 363, 603 (1993).

[2] E.B. Barros, H. Son, Ge.G. Samsonidze, A.G. Souza Filho, R. Saito, Y.A. Kim, H. Muramatsu, T. Hayashi, M. Endo, J. Kong, M.S. Dresselhaus, Phys. Rev. B 76, 045425 (2007).

[3] A.G. Souza Filho, V. Meunier, M. Terrones, B.G. Sumpter, E.B. Barros, F. Villalpando-Paez, J.M. Filho, Y.A. Kim, H. Muramatsu, T. Hayashi, M. Endo, M.S. Dresselhaus, Nano Lett. 7, 2383 (2007).

[4] A.G. Souza Filho, M. Endo, H. Muramatsu, T. Hayashi, Y.A. Kim, E.B. Barros, N. Akuzawa, Ge.G. Samsonidze, R. Saito, M.S. Dresselhaus, Phys. Rev. B 73, 235413 (2006).

[5] S.D. Kim, J.W. Kim, J.S. Im, Y.H. Kim, Y.S. Lee, J. Fluorine Chem. 128, 60 (2007).
[6] F. Borondics, K. Kamaras, M. Nikolou, D.B. Tanner, Z.H. Chen, A.G. Rinzler, Phys. Rev. B 74, 045431 (2006).

[7] W. Zhou, J. Vavro, N.M. Nemes, J.E. Fisher, F. Borondics, K. Kamaras, D.B. Tanner, Phys. Rev. B 71, 205423 (2005).

[8] R. Graupner, J. Abraham, A. Vencelova, T. Seyller, F. Hennrich, M.M. Kappes, A. Hirsch, L. Ley, Phys. Chem. Chem. Phys. 5, 5472 (2003).

[9] J. Cambedouzou, J.L. Sauvajol, A. Rahmani, E. Flahaut, A. Peigney, C. Laurent, Phys. Rev. B 69, 235422 (2004).

[10] T. Saito, K. Matsushige, K. Tanaka, Physica B 323, 280 (2002).

[11] S.-M. Yuen, C.-C. Ma, Y.-Y. Lin, H.-C. Kuan, Composit. Sci. Technol. 67, 2564 (2007).

[12] L. Duclaux, Carbon 40, 1751 (2002).

[13] A. Bachmatiuk, E. Borowiak-Palen, R.J. Kalenczuk, Nanotechnology 19, 365605 (2008).

[14] E. Kowalska, P. Kowalczyk, J. Radomska, E. Czerwosz, H. Wronka, M. Bystrzejewski, J. Thermal Anal. Calorim. 86, 115 (2006).

[15] A.M. Rao, S. Bandow, E. Ritchter, P.C. Eklund, Thin Solid Films 331, 141 (1998).

[16] J. Fisher, Acc. Chem. Res. 35, 1079 (2002).

[17] S. Costa, A. Bachmatiuk, E. Borowiak-Palen, R. Kalenczuk, Pol. J. Chem. Tech. 10, 34 (2008).

[18] A. Jorio, M.A. Pimenta, A.G. Souza Filho, R. Saito, G. Dresselhaus, M.S. Dresselhaus, New J. Phys. 5, 139.1 (2003).

[19] S.B. Fagan, A.G. Souza Filho, J. Mendes Filho, P. Corio, M.S. Dresselhaus, Chem. Phys. Lett. 406, $54(2005)$. 